\title{
Ion beam assisted crystallization of amorphous silicon layers using high current density Gallium beams
}

\author{
Jonathan England ${ }^{\mathrm{a}, *}$, Michael W. Phaneuf $^{\mathrm{b}}$, Alexandre Laquerre ${ }^{\mathrm{b}}$, Andrew Smith ${ }^{\mathrm{c}}$, Russell Gwilliam ${ }^{\mathrm{c}}$ \\ a Varian Semiconductor Equipment Associates, 35 Dory Road, Gloucester, MA, 01930, USA \\ ${ }^{\mathrm{b}}$ Fibics Incorporated, 556 Booth Street, Suite 200, Ottawa, Ontario, K1A 0G1, Canada \\ c University of Surrey, Guildford, Surrey Gu2 7XH, UK
}

\section{A R T I C L E I N F O}

\section{Article history:}

Available online $\mathrm{xxxx}$

\begin{abstract}
A B S T R A C T
We have investigated ion beam assisted crystallization as a possible method for the creation of crystalline Si layers on amorphous substrates. Ion beam assisted crystallization has previously been characterised with pulsed and modest continuous beam current densities, but not at the high densities that would be required for high throughput processes. In this study, amorphous Si layers were implanted with Ga using a focused ion beam (FIB) tool and with $\mathrm{Ga}, \mathrm{Ar}$, Ge and As using beamline implanters. Crystallization was only observed for Ga, implying Ga alone was acting to reduce the activation energy. The observed rates of crystallization also implied that the relaxation state of the a-Si, influenced by the beam conditions, was an important driver for the phase transformation.
\end{abstract}

(c) 2011 Elsevier B.V. All rights reserved.

\section{Introduction}

Crystallization using heat sources of various thermal budgets has been used in semiconductor processing for many years. Furnaces, rapid thermal annealers, flash lamps and lasers have been used to re-grow crystal layers that have been damaged during processing by ion implantation. Ion beam annealing is also known [1]. Ion collisions can break atomic bonds in the damaged layer near to the underlying crystalline interface which can re-connect onto atoms in the crystalline substrate, leading to solid phase epitaxial regrowth. Ion collisions can also cause damage in the crystalline layer, causing amorphisation, but with the correct choice of beam conditions, net crystallization can occur [2].

Technologies such as flat panel displays and thin solar cells require crystallization of amorphous silicon (a-Si) that has been deposited onto substrates which can only tolerate modest temperatures (typically less than $600^{\circ} \mathrm{C}$ for glass). In this case, there is no dominating amorphous/crystalline interface on which to regrow, and crystallization has to proceed through a nucleation phase followed by growth of the nuclei. Crystallization of the a-Si can be achieved thermally. Solid phase crystallization for solar cells has been achieved using long $(9 \mathrm{~h})$, low temperature $\left(500^{\circ} \mathrm{C}\right)$ furnace anneals [3]. For flat panel displays, the processes of record, excimer laser annealing [4], uses pulsed lasers with a typical power density of $3 \times 10^{10} \mathrm{~W} / \mathrm{m}^{2}$ to melt the a-Si layer (melting point $\sim 1000^{\circ} \mathrm{C}$

\footnotetext{
* Corresponding author.

E-mail address: jonathan.england@vsea.com (J. England).
}

[5]). The pulsed nature of the beam means that only the surface a-Si layer is melted whilst the glass substrate remains relatively cool (see Fig. 1). Crystallization can also be driven by particle bombardment, and the use of ion beams could have several potential advantages. The use of non-equilibrium processes (i.e. bond breaking using ion collisions rather than temperature) may allow access to novel process mechanisms. Many ion beam characteristics (energy, species, dose, dose rate and beam profile) could be varied for process optimisation, and ion beams are often more controllable and repeatable than laser beams. However, sputtering and unwanted consequences of the ions implanted in the crystallised material are potential disadvantages. Pulsed electron [6] and pulsed ion beams [7] have been used to melt the a-Si and keep the substrate cool. Solid phase crystallization using continuous ion beams at low fluences has also been extensively studied, but the conditions necessary for crystallization implies that the crystallization rate would be too slow to be viable as an industrial process. Thermal modelling suggested that a continuous ion beam of with a power density of $1.7 \times 10^{7} \mathrm{~W} / \mathrm{m}^{2}$ scanned over a substrate at realistic velocities could melt an a-Si layer without overly heating the substrate (Fig. 1). Liquid phase (ion beam assisted) crystallization could then proceed. However, if the beam could only be focused down into a $1 \mathrm{~mm}$ wide ribbon, it would take too long (2.4 s) to melt the a-Si. The whole substrate would overheat (the bottom of the glass sheet would reach $600{ }^{\circ} \mathrm{C}$ ) and the process throughput would be too low [8].

This study investigated if achievable continuous, high intensity ion beams could be used to crystallise amorphous silicon layers to a sufficient quality and at rates that could be of industrial interest. 


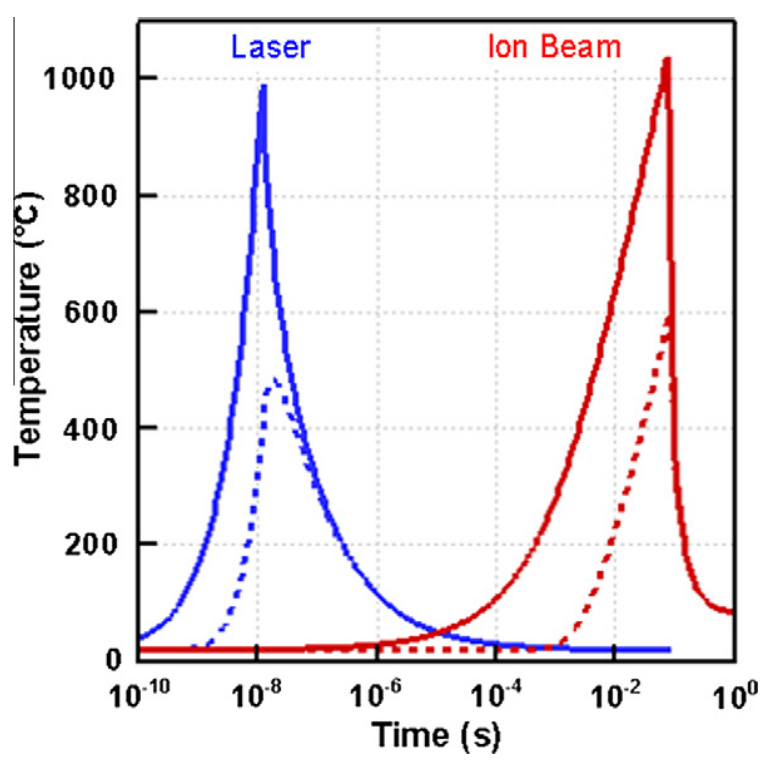

Fig. 1. Calculated temperature temporal profiles for a $50 \mathrm{~nm}$ thick a-Si layer (solid lines) on glass, heated using a single excimer laser pulse $\left(360 \mathrm{~mJ} / \mathrm{cm}^{2}\right.$ for $12 \mathrm{~ns}$ in a beam $465 \mathrm{~mm}$ by $0.4 \mathrm{~mm}$ ) and a $20 \mathrm{keV}$ Ar ribbon ion beam ( $25 \mathrm{~mA}$ focused into a ribbon of $300 \times 0.1 \mathrm{~mm}$, dwelling for $80 \mathrm{~ms}$ ). Temporal temperature profiles are shown for the glass substrate (dotted lines). For the pulsed laser case (profile $0.1 \mu \mathrm{m}$ below surface shown), the temperature remains below $600{ }^{\circ} \mathrm{C}$ close to its surface. For the ion beam case (profile $50 \mu \mathrm{m}$ below surface shown), the temperature for the top $50 \mu \mathrm{m}$ exceeds $600{ }^{\circ} \mathrm{C}$.

\section{Experiment and results}

\subsection{FIB implants}

Because a highly focused ribbon beam was not immediately available, the possibility of high current density ion assisted crystallization was first investigated using a focused spot beam which could be generated in a FIB. This however dictated that the first ion species to be investigated was Ga. The dual column Zeiss NVision FIB with integrated Gemini FE-SEM at Fibics Inc had the advantage that it allowed real time in situ imaging of the experiments. The FIB column was tuned to give $30 \mathrm{keV}$ Ga beams with uniform circular cross sections and of varying current densities as summarised in Table 1.

The first substrates investigated consisted of $50 \mathrm{~nm}$ thick a-Si deposited on quartz. To avoid surface charging in the FIB, these samples were coated with a layer of $\mathrm{Au} / \mathrm{Pd}$, which was removed from the area to be implanted. Series of beam burns at different dwell times were produced for each beam current density. SEM images showed "pie crust" features suggesting the onset of crystallization after dwell times depending on the beam current density (Fig. 2). Crystallization was later seen in TEM cross sections
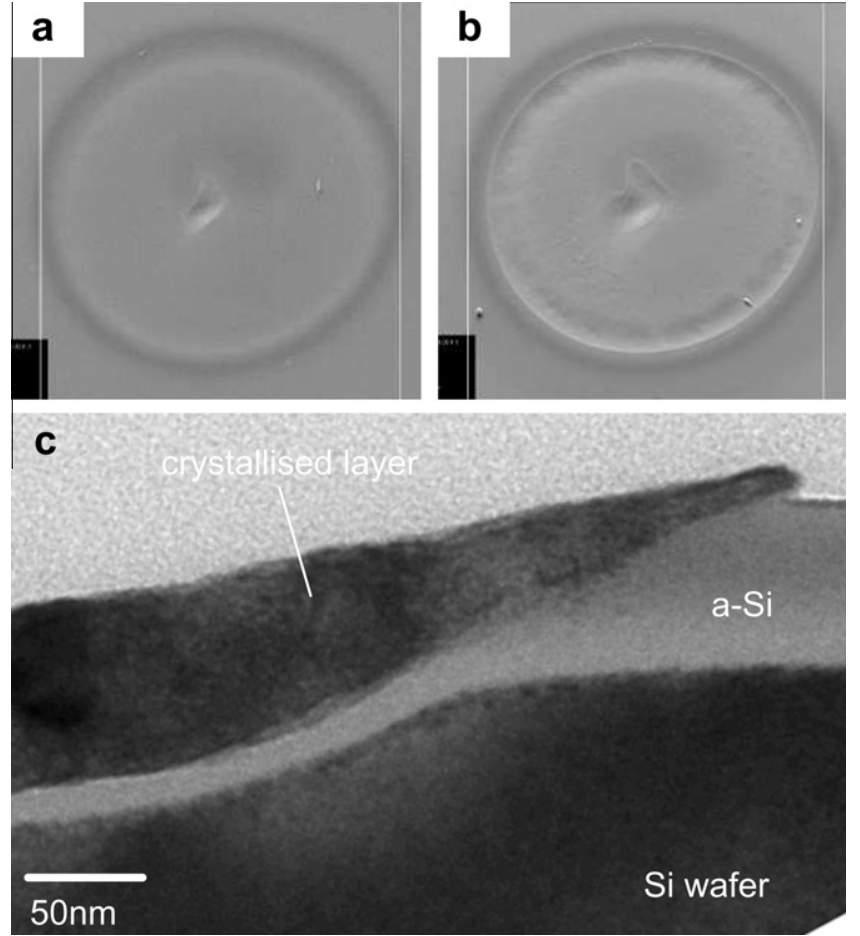

Fig. 2. (a) Top down SEM views of FIB beam $\left(5.5 \mathrm{~mA} / \mathrm{cm}^{2}\right)$ burns after dwell times of (a) $1 \mathrm{~s}$ (still amorphous) and (b) $2 \mathrm{~s}$ (after crystallization has occurred). The white vertical lines are $25 \mu \mathrm{m}$ apart. (c) A bright field TEM cross section at the edge of the "pie crust" showing crystallization has occurred for a FIB beam of $5.5 \mathrm{~mA} / \mathrm{cm}^{2}$ in the amorphous layer, unconnected to the substrate.

(Fig. 3). When fabricating TEM lamellae using the FIB, a thick capping layer (e-beam deposited carbon) was first deposited on the sample and the lamellae were not machined as thinly as normal to avoid the possibility that artifacts could be introduced by the milling beam.

Sputtering at the beam centre in the early experiments (such as Fig. 3) demonstrated the need to carefully adjust the FIB column to produce beams of uniform current densities. The beam profile uniformity could be measured by observing the uniformity of the depth profile of a sputter crater left when the beam was dwelled onto the surface of a Si wafer. Wafers were convenient to process as they did not exhibit any surface charging. The surface of a test wafer was amorphised using a scanned low current density Ga beam in the FIB. A series of beam burn experiments carried out on these samples were found to require the same conditions to cause crystallization as on the a-Si deposited on quartz samples. The beam conditions are summarised in Table 1 .

For a later series of measurements, an Oxford Instruments EBSD (electron back scattered diffraction) system had been installed

Table 1

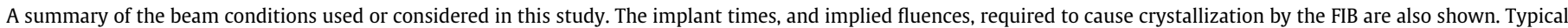
beam conditions used in the previous studies of ion beam assisted solid state crystallization are included for comparison.

\begin{tabular}{|c|c|c|c|c|}
\hline Nominal beam condition & $\begin{array}{l}\text { Beam density } \\
\left(\mathrm{mA} / \mathrm{cm}^{2}\right)\end{array}$ & $\begin{array}{l}\text { Fluence rate } \\
\text { (ions } / \mathrm{cm}^{2} / \mathrm{s} \text { ) }\end{array}$ & $\begin{array}{l}\text { Implant time to } \\
\text { crystallise (s) }\end{array}$ & $\begin{array}{l}\text { Fluence to crystallise } \\
\left(\text { ions } / \mathrm{cm}^{2}\right)\end{array}$ \\
\hline FIB (Ga, $3 \mathrm{nA}$ into1.7 $\mu \mathrm{m}$ diameter spot) & 130 & $8 \times 10^{17}$ & 0.06 & $4.9 \times 10^{16}$ \\
\hline $\mathrm{FIB}(\mathrm{Ga}, 3 \mathrm{nA}$ into $3 \mu \mathrm{m}$ diameter spot) & 50 & $3 \times 10^{17}$ & 0.15 & $4.7 \times 10^{16}$ \\
\hline FIB (Ga, $27 \mathrm{nA}$ into $25 \mu \mathrm{m}$ diameter spot) & 5.5 & $3 \times 10^{16}$ & 2 & $6.9 \times 10^{16}$ \\
\hline FIB (Ga, $27 \mathrm{nA}$ into $65 \mu \mathrm{m}$ diameter spot) & $0.6-1.2$ & $4-7 \times 10^{15}$ & $10-25$ & $0.7-1 \times 10^{17}$ \\
\hline Danfysik implanter (Ga, As, Ar, $7.5 \mu \mathrm{A}$ into $10 \mathrm{~mm}$ diameter static beam) & 0.1 & $6 \times 10^{14}$ & & \\
\hline Vista 80 , focused $(25 \mathrm{~mA} ; 300 \times 0.1 \mathrm{~mm})$ & 67 & $4 \times 10^{17}$ & & \\
\hline Vista 80 , standard (Ge, Ar, $20 \mathrm{~mA} ; 300 \times 20 \mathrm{~mm})$ & 0.3 & $2 \times 10^{15}$ & & \\
\hline Previous studies (many species) [2] & 0.001 & $7 \times 10^{12}$ & & $2.2 \times 10^{16}$ \\
\hline
\end{tabular}



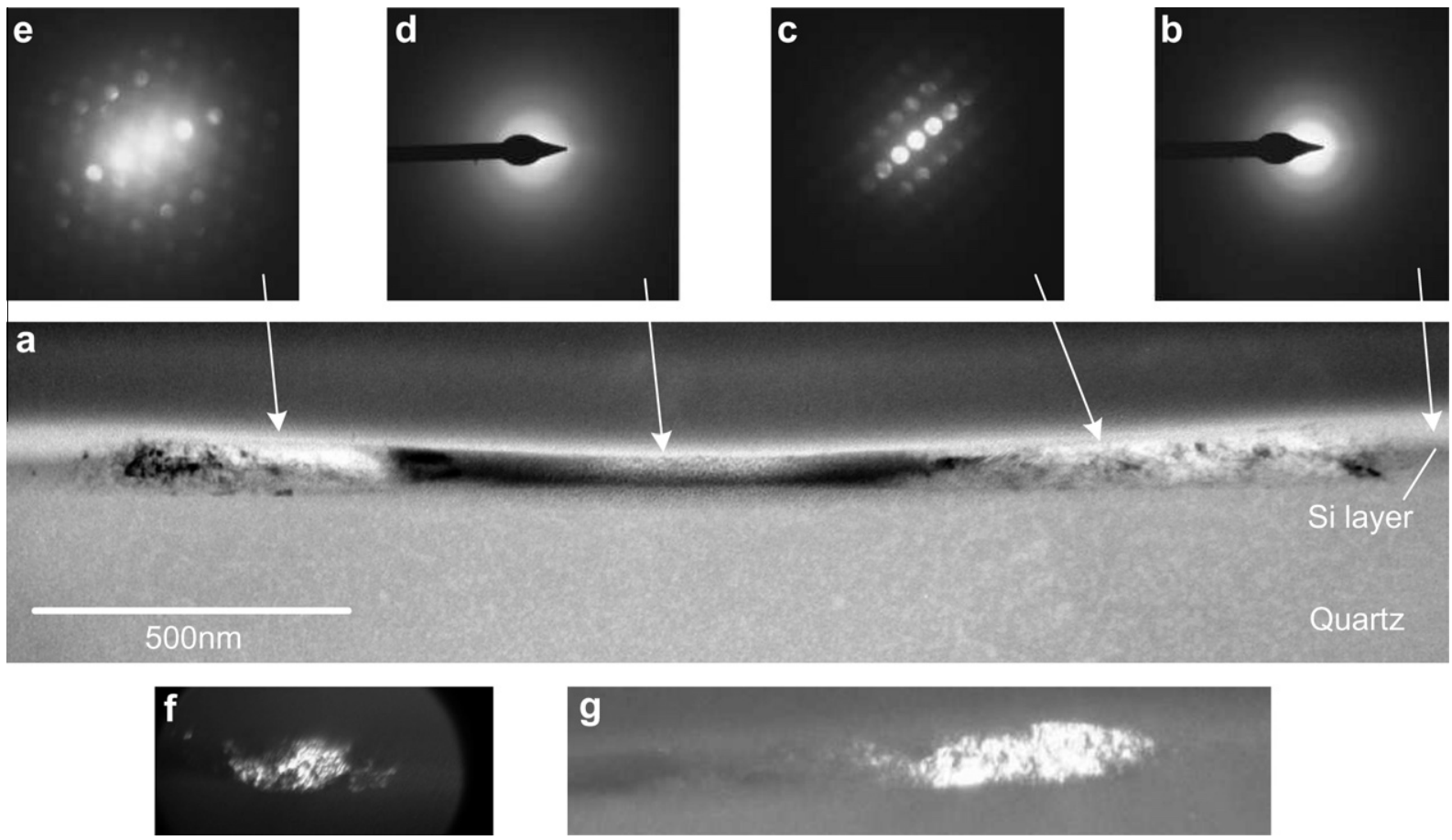

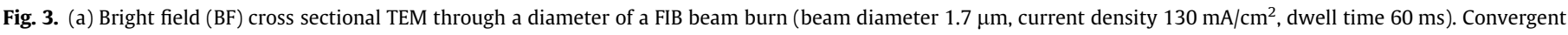

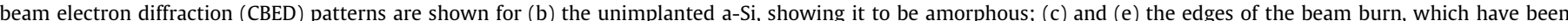

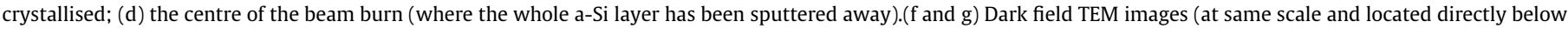
their positions in the BF image) at the edges of the beam burn using electrons scattered in the directions of the brightest CEBD spots.

onto the SEM, allowing real time in situ measurement of crystallinity. EBSD measurements showed that, surprisingly, crystallization did not actually occur during the implant, but had occurred before the samples were re-measured some $12 \mathrm{~h}$ after the implants had been completed. EBSD showed that the orientation of the crystals produced in the amorphised Si wafers were oriented differently than the substrate, and TEM cross sections showed that crystallization had not originated on the crystalline substrate (Fig. 2). A further surprise was that calculations showed that the temperature rise for the a-Si under a FIB beam was only $80^{\circ} \mathrm{C}$ due to fast thermal diffusion in all radial directions away from a small spot, rather than just in the two directions when considering a ribbon beam.

\subsection{Beamline implants}

A $200 \mathrm{keV}$ capable Danfysik 1090 ion implanter was used at The University of Surrey to study Ga implants at lower current densities. Pieces of a Si wafer, pre-amorphised by implants of $100 \mathrm{keV}$ Si to $1 \times 10^{17}$ ions $/ \mathrm{cm}^{2}$, were implanted with 30,35 and $120 \mathrm{keV}$ $\mathrm{Ga}$ to fluences of around $2 \times 10^{17}$ ions $/ \mathrm{cm}^{2}$. The beams were parked (rather than being electrostatically scanned as usual) on a static sample to allow the implants to be completed in $\sim 8 \mathrm{~min}$. A sample could be mounted in poor thermal contact with the sample holder to allow self heating from the beam or better contacted and cooled or heated using a backside, resistive heater. The samples' front-side temperatures were monitored during the implants using a thermocouple. After an implant had been completed, the sample was removed from the vacuum chamber and walked to a neighbouring laboratory so that Raman spectroscopy could be carried out. Crystallization was only observed when the substrate exceeded $188^{\circ} \mathrm{C}$ during the implants. Fig. 4 shows an example of how the crystal sizes and heights of the characteristic c-Si peak at $\sim 520 / \mathrm{cm}$ in Raman spectra decreased as the distance from the beam centre increased. The crystal sizes increased for higher sub-
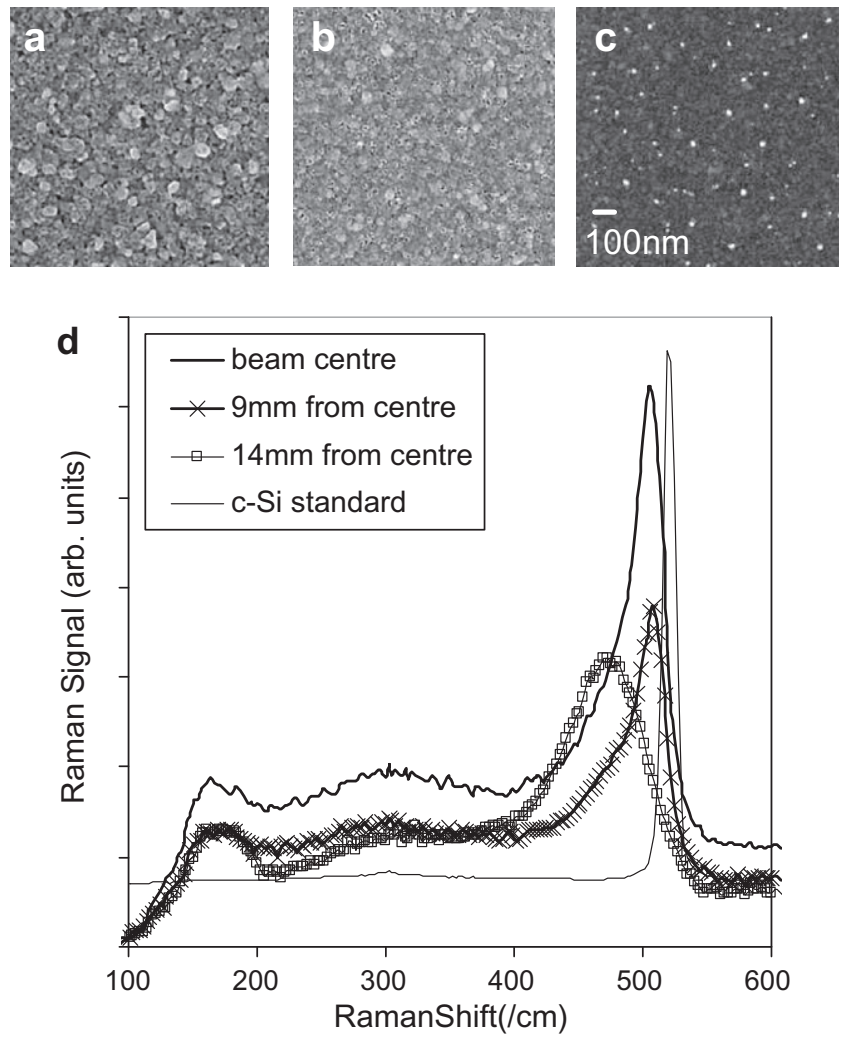

Fig. 4. SEM images of the substrate after a static beamline implant ( $35 \mathrm{keV} \mathrm{Ga}$ to $2.7 \times 10^{17}$ ions $/ \mathrm{cm}^{2}$ at a temperature of $280^{\circ} \mathrm{C}$ ) at (a) the centre of the beam burn, (b) $9 \mathrm{~mm}$ and (c) $14 \mathrm{~mm}$ from the centre. (d) Raman spectra taken at these positions, compared to a c-Si standard. 
strate temperatures, and heated implants of $120 \mathrm{keV} \mathrm{Ga} \mathrm{produced}$ larger crystals than the lower energy implants. Raman spectra measured for regions of the samples protected from the ion beam (underneath the thermocouple) were identical to spectrum (c) and comparable to an a-Si standard. Samples that had not crystallised were re-measured by Raman spectroscopy a few weeks later and then again after spending $24 \mathrm{~h}$ in an oven at $175^{\circ} \mathrm{C}$, but were still found to be amorphous. Implants of $30 \mathrm{keV}$ As and Ar were also carried out under similar conditions, but failed to cause any crystallization.

Implants of $30 \mathrm{keV}$ Ge up to fluences of $5 \times 10^{17} \mathrm{ions} / \mathrm{cm}^{2}$ into $50 \mathrm{~nm}$ a-Si deposited on $80 \mathrm{~nm}$ oxide coated wafers and $20 \mathrm{keV}$ Ar to $2 \times 10^{17}$ ions $/ \mathrm{cm}^{2}$ into pre-amorphised wafers (see above) were carried out at Varian Semiconductor Equipment Associates, Gloucester using a Vista 80 high current implanter (using a standard, non focused ion beam) but also failed to produce crystallization when measured a few days later by cross sectional TEM and Raman Spectroscopy at Cerium Laboratories.

\section{Discussion}

Before crystallization can proceed, an activation barrier, $\Delta G_{\text {act }}$ in Fig. 5, has to be overcome. If crystallization is activated thermally, the rate for overcoming the barrier will be increases if $\Delta G_{\text {act }}$ is reduced, or the temperature increased. The observation that crystallization only occurred for Ga suggests that Ga was acting as a catalyst to reduce $\Delta G_{\text {act }}$. The enhancement of crystallization by metal catalysis (or silicide formation) has been exploited in the MILC (metal induced lateral crystallization) and ALLILE (Aluminium induced layer exchange) processes [9]. a-Si is known to have a higher Gibbs free energy than c-Si, shown as $\Delta G$, in Fig. 5 . The magnitude of $\Delta G$ depends on the state ("relaxation") of the a-Si. Highly damaged a-Si will be highly un-relaxed. The relaxation a$\mathrm{Si}$ is expected to increase for lower dose rates and higher substrate temperatures [5]. Thermodynamically, crystallization becomes more favourable as $\Delta G$ increases. The affect on crystallization rate could be extremely strong. Im and Atwater calculated that the rate of nucleation in beam assisted crystallization could vary by seven orders of magnitude if $\Delta G$ changed by $10 \%$ [10]. We suggest that the high density FIB beams implanting substrates at $<80^{\circ} \mathrm{C}$ had created highly un-relaxed a-Si. Although the crystallization rate was not high enough for significant crystallization to occur over the time that the beam was present (up to $25 \mathrm{~s}$ ), crystallization could proceed over a few hours at room temperature. An increased

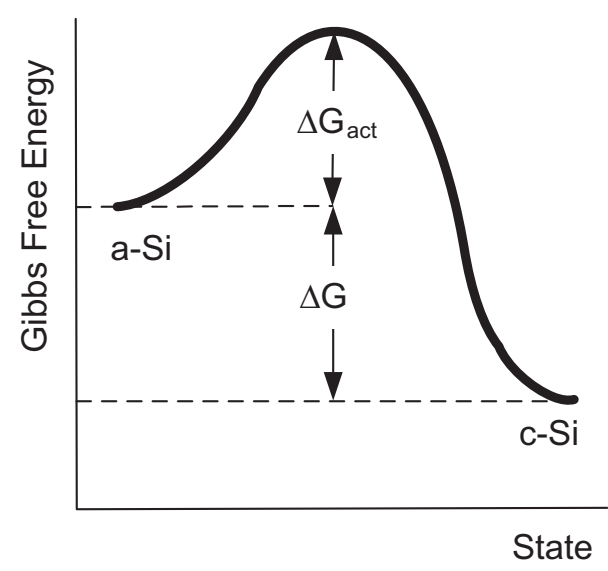

Fig. 5. A phase change diagram showing the meaning of $\Delta G_{\text {act }}$, the activation energy, and $\Delta G$, the "driving force" of the crystallization phase transformation (after [9]).
$\Delta G$ at higher fluence rates may also explain why the more focused FIB beams required smaller doses to cause crystallization than the larger FIB beams (see Table 1).

For the lower current density beams generated in the beamline implanters, we suggest that the a-Si was more relaxed (smaller $\Delta G)$. Indeed, Im and Atwater explain that in the regime where dynamic annealing takes place (such as the lower fluence, high substrate temperature implants at The University of Surrey) crystal nucleation may only take place in the early part of the damage cascade before dynamic annealing has occurred. Hence crystallization would only proceed when the beam was present, as observed in these experiments. Even then, the reaction rate was not high enough to cause significant crystallization during the implant (minutes) unless the substrates were heated. This observation suggests that the increased rate of overcoming $\Delta G_{\text {act }}$ at the higher temperatures must have had a stronger effect on the crystallization rate than that caused by any reduction in $\Delta G$ caused by increased self annealing. The observations of reduced crystal size and crystallinity where the beam current density decreased, and the larger crystal sizes for higher energy implants can also be explained by the dose rate effect on $\Delta G$.

The unusual behaviour of the Ga-Si eutectic may also play a role. Several studies have reported Ga segregation effects in thermally and ion beam annealed Ga doped Si $[11,12]$. Ga has a low solid solubility in Si. The eutectic temperature is only slightly higher than room temperature $\left(29.8^{\circ} \mathrm{C}\right)$ and the eutectic composition is almost pure Ga (99.994 atom\% Ga:0.006 atom\% Si) [13]. Any local heating by the beam or exothermic reaction could lead to the formation of liquid Ga. Evidence of Ga flow was observed on TEM samples that were re-measured 60 days after the experiments had been completed.

\section{Conclusions}

Ga was the only species seen to cause crystallization under the beam current density conditions of this study, suggesting Ga acted as a catalyst for crystallization. We suggest that the high Ga FIB current density left the a-Si in an un-relaxed state, which allowed the transition to c-Si to occur around room temperature over a period of hours after the implant was completed. For the lower implant current densities of the beamline implanter, we suggest that the a-Si was more relaxed; and crystallization could only proceed during the implant and when the substrate was heated.

The ability to measure crystallinity in situ allowed important observations as to when crystallization occurred in the FIB. These results will be of particular interest to analysts who use FIB beams to create lamellae for TEM analyses as we have shown that care must be taken so that the FIB beam does not cause crystallization in amorphous samples.

\section{Acknowledgements}

The authors acknowledge the help of Rajesh Dorai for the thermal modelling of laser and ion beams into substrates; Vlad Stolojan at Surrey University for additional TEM measurements and Cerium Laboratories, Austin, TX, USA, for additional TEM and Raman spectroscopy.

\section{References}

[1] Chen, J. Wu, Y. Yang, K. Hsieh, M. Lin, R. Huang, J. Appl. Phys. 52 (1981) 3304

[2] C. Spinella, S. Lombardo, F. Priolo, J. Appl. Phys. 84 (1998) 5383.

[3] Schneider, R. Evans, Proc. 21st EUPVSEC, Dresden (2006), 1032.

[4] US patent $7259,081$.

[5] Pelaz, L. Marques, J. Barbolla, J. Appl. Phys. 96 (2004) 5947.

[6] A. Greenwald, A. Kirkpatrick, R. Little, J. Minnucci, J. Appl. Phys. 50 (1979) 783. [7] R. Fastow, J. Mayer, J. Appl. Phys. 61 (1987) 175. 
[8] US patent application 20090124064

[9] J. Schneider, Nucleation and growth during the formation of polycrystalline silicon thin films, Ph.D. thesis, Technischen Universität Berlin, 2005.

[10] J. Im, H. Atwater, Nucl. Instr. and Meth. B59-60 (1991) 422.
[11] S. Withrow, O. Holland, S. Pennycook, Appl. Surf. Sci. 43 (1989) 191

[12] R. Elliman, G. Carter, Nucl. Instr. and Meth. 209-210 (1983) 663.

[13] R. Olesinski, N. Kanani, G. Abbaschian, in: T. Massalski, W.W. Scott Jr. (Eds.), Binary Alloy Phase Diagrams, Vol. 2, 1990, p. pp. 1856. 